\title{
PEPCONTEXTUAL: definição de um prontuário eletrônico de paciente ciente de contexto
}

\author{
William Hart Oliveira ${ }^{1}$ \\ Cristiano André da Costa ${ }^{1}$ \\ Rodrigo da Rosa Righi ${ }^{1}$ \\ Dinarte Alexandre Ballester ${ }^{2}$
}

\begin{abstract}
Resumo: A computação móvel pode ser definida como informação na ponta dos dedos a qualquer hora, em qualquer lugar e avança como uma realidade nas tarefas e atividades das pessoas, em decorrência da popularização e diversificação de dispositivos móveis e redes sem fio. Faz-se importante então, desenvolver modelos que permitam não somente compartilhar dados clínicos, mas sim tornar os dados independentes da tecnologia usada e cientes de contexto. Neste cenário, o presente trabalho, denominado PEPContextual, consiste em um modelo que faz uso da ciência da situação (situation awareness), explorando informações relacionadas com o ambiente ou com os próprios usuários e onde diversos tipos de contextos são aglomerados de forma a gerar uma visualização mais rica, complexa e inteligente, criando inúmeras possibilidades, dentre elas, a inferência de riscos associados ao paciente. A avaliação por estudo de caso confirmou a expectativa de que a aplicação de ciência de situação, baseada no modelo de Endsley, possibilitaria que o modelo de forma ubíqua detectasse riscos associados ao paciente.
\end{abstract}

\section{Palavras-chave: Ciência de Situação. Prontuário Eletrônico. Saúde Ubíqua.}

\begin{abstract}
Ubuiquitous computing can be defined as information at anytime, anywhere and its grows as a reality on people's activities and daily tasks through the diversification and popularization of mobile devices and networks. So, it's important create models that allow not only share clinical data, but increase its quality, making it indifferent to technology and context aware. In this case, the present paper, called PEPContextual, is about a model that makes use of situation awareness, exploring environment related information and/or its own users, where several types of contexts are combined looking a richer, complex and smart visualization, creating several possibilities and, among that, the inferrence of associated risks to patients. The evaluation by case study has confirmed the proposal that situation awareness, based on Endsley model, makes possible that the model is capable to find associated risks through ubiquity.
\end{abstract}

Keywords: Context Awareness. Situation Awareness. Ubiquitous Computing. Eletronic Health Record. Ubiquitous Health

\section{Introdução}

O surgimento de novas tecnologias da informação no cotidiano possibilitou diversas formas de comunicação e obtenção de informação. Estudos sobre mobilidade em sistemas distribuídos vêm sendo impulsionados pela proliferação de dispositivos móveis e pela exploração de novas tecnologias baseadas em

\footnotetext{
${ }^{1}$ Programa de Pós-Graduação em Computação Aplicada (PIPCA), Universidade do Vale do Rio dos Sinos (Unisinos), São Leopoldo (RS) - Brasil

\{william.hart.oliveira@gmail.com, cac@unisinos.br, rrighi@unisinos.br\}

${ }^{2}$ Sistema de Saúde Mãe de Deus, Gerência dos Serviços de Saúde Mental, Avaliação de Risco do Comportamento, Porto Alegre (RS) - Brasil

\{dinarte.ballester@maededeus.com.br\}
}

http://dx.doi.org/10.5335/rbca.v9i2.6149 
redes sem fio [1]. A computação móvel pode ser definida como informação na ponta dos dedos a qualquer hora, em qualquer lugar e avança como uma realidade nas tarefas e atividades das pessoas, em decorrência da popularização e diversificação de dispositivos móveis e redes sem fio [2]. Como consequência, nasce o suporte computacional contínuo, denotando o conceito de computação ubíqua [3, 4].

Com isso, outro tema relacionado é o conceito de ciência de contexto (context awareness), que aliada à propagação da comunicação sem fio permitiu aos serviços computacionais serem disponibilizados em contextos específicos [5]. A ciência de contexto consiste na capacidade de o sistema reagir a dados externos que podem influenciar uma aplicação [6]. Além disso, proporciona certa inteligência ao software com o intuito de ajudar a fornecer informações e tomar decisões baseadas nas preferências do usuário ligadas a um contexto como localização, preferências pessoais, dentre outras [7, 8].

Uma possibilidade nesse sentido, decorrente da disseminação dos dispositivos móveis e do crescimento no surgimento de sensores corporais (wearable computing), é permitir que o paciente interaja com o seu PHR (Personal Health Record) a partir de múltiplos dispositivos móveis. Estes acessos podem ser combinados ou não com informações armazenadas de indicadores [9] decorrentes de sensores corporais - a chamada saúde ubíqua (ubiquitous health) - utilizados em sistemas de saúde EHR (Electronic Health Record) baseados na ciência da situação (situation awareness) [10].

A saúde ubíqua consiste no uso da ciência de contexto sobre elementos do ambiente e dos usuários, inseridos neste de forma proativa, através da combinação de interfaces intuitivas e processos onde os usuários não necessitam de conhecimentos específicos para utilizar os sistemas de saúde de forma plena [11]. Já a ciência da situação significa conhecer tudo sobre o que está acontecendo no escopo de uma situação, ligado ao conhecimento de um evento ou fenômeno em particular [12].

Faz-se importante então, desenvolver modelos que permitam não somente compartilhar dados clínicos, mas sim aumentar a longevidade dos dados, melhorar a sua qualidade, tornar os dados independentes da tecnologia usada e cientes de contexto. Neste cenário, o presente trabalho, denominado PEPContextual, consiste em um modelo que faz uso da ciência de contexto $[6,13]$ e ciência da situação (situation awareness), explorando informações relacionadas com o ambiente ou com os próprios usuários e onde diversos tipos de contextos são aglomerados de forma a gerar uma visualização mais rica, complexa e inteligente, criando inúmeras possibilidades, dentre elas, a inferência de riscos associados ao paciente $[14,15]$.

O uso atual de um PHR é a agregação das informações obtidas pelo próprio paciente, como dados coletados através de sensores de um smartphone e com as informações do prontuário eletrônico [19]. Entretanto, os PHRs permitem que o paciente obtenha o direito sobre seus dados, como o histórico médico, sintomas, informações relacionadas à saúde da pessoa, entre outros [20]. A participação do paciente na interação com seu prontuário deve sofrer mudanças em vista da utilização dos dispositivos móveis e a captura de indicadores através de sensores, além de agregar valor às informações e tornar possível uma ação mais proativa [7].

A integração das áreas de saúde ubíqua, abrangendo o uso do prontuário pessoal do paciente, nasce da evolução do acesso à informação a partir dos dispositivos móveis e da mobilidade destes equipamentos e suas integrações com múltiplos sensores [10]. Neste sentido, acabam surgindo diversos desafios de pesquisa, como a compatibilidade com diferentes plataformas e como lidar com o excesso de informações [7].

Uma aplicação ciente de situação interage com o usuário, aprende com o comportamento, suas ações obtidas através de sensores físicos e virtuais - e autonomamente sugere novas ações e/ou infere situações de acordo com o contexto situacional atual [14]. O modelo proposto consiste em um prontuário eletrônico do paciente focado em dispositivos móveis, no âmbito da saúde ubíqua que utiliza a ciência da situação e permita que os provedores de saúde possam agir de forma proativa.

O principal objetivo deste trabalho é desenvolver um modelo ubíquo chamado PEPContextual, que faz uso da ciência da situação, explorando informações relacionadas com o ambiente ou com os próprios usuários e onde diversos tipos de contextos são aglomerados de forma a inferir riscos associados à saúde dos pacientes.

O texto está organizado em 6 seções. O presente trabalho está organizado na seguinte sequência. A seção 2 apresenta os conceitos básicos que são essenciais para a compreensão do modelo proposto. A seção 3 descreve em detalhes o modelo que envolve a inferência de riscos associados através das informações do PHR de acordo com o contexto e situação do paciente. A seção 4 apresenta a implementação do protótipo que representa o modelo, bem como as ferramentas e metodologias aplicadas, com amostras da aplicação para o dispositivo do profissional e servidor, este último responsável pela inferência e análise de riscos associados. A seção 5 descreve 
a avaliação aplicada ao modelo proposto, bem como os cenários propostos e avaliação dos mesmos, bem como da usabilidade do protótipo. Para finalizar, são apresentados na seção 6 o desfecho e as contribuições desta pesquisa.

\section{Referencial Teórico}

Nesta seção procura-se abordar aspectos teóricos sobre os conceitos de computação móvel, ubíqua, saúde ubíqua, contexto, situação, entre outros.

\subsection{Computação Ubíqua}

A computação ubíqua é definida como um ambiente de computação altamente distribuído e integrado, recheado de dispositivos computacionais onde a percepção de PC (personal computer) que conhecemos não prevalece [4]. Sudha defende que um grande número de agentes computacionais e dispositivos de comunicação que trabalham em conjunto caracterizam a computação ubíqua [27]. Ainda, Weiser definiu que o relacionamento entre as pessoas e os computadores se tornaria transparente, isto é, as ações aconteceriam da forma mais natural possível, sem que o usuário perceba que está acionando um dispositivo ou uma aplicação $[4,28]$.

A computação móvel envolve os sistemas computacionais nos mais diversos dispositivos trocando informações através de redes sem fio, permitindo a mobilidade. Na computação ubíqua os dispositivos móveis conseguem contextualizar as aplicações, adequando-se ao usuário de acordo com os serviços distribuídos na rede, possibilitando a realização de tarefas sem levar em conta a tecnologia. Já a computação pervasiva é a composição de cinco áreas de pesquisa - computação móvel, rede wireless, computação embarcada, ciência de contexto e IHC (Human Computer Interaction) [29].

\subsection{Ciência de Contexto e Ciência da Situação}

Outra característica da computação ubíqua é ciência de contexto. A aplicação dita ciente de contexto coleta elementos do ambiente e dos usuários inseridos neste e a partir deste ponto diversas adaptações podem ser realizadas de forma proativa por sistemas ubíquos. Estas modificações ajudam na realização das tarefas do usuário, pois através da combinação de interfaces intuitivas e processos proativos baseados em contexto, os usuários não precisam de conhecimentos específicos de computação para utilizar os sistemas de saúde de forma plena [11].

A definição de aplicações cientes de contexto divide-se em duas categorias: as que se utilizam de informações de contexto e as que adaptam essas informações [30]. Além disso, definem-se aplicações cientes de contexto como adaptáveis à localização, pessoas, objetos próximos e as mudanças ocorridas destas adaptações com o passar do tempo [2].

Para isso, uma aplicação móvel ciente ao contexto tem de ser capaz de extrair do ambiente, usuário ou tempo, dados que ajudem na realização da tarefa [31]. Também, capturar de maneira ágil os serviços que estejam na rede para que o usuário possa utilizá-los na realização da tarefa escolhida. Tudo que esteja inserido no ambiente é contexto, desde que seja relevante para a aplicação. Nesse sentido, a saúde ubíqua é um campo emergente de tecnologia que utiliza um grande número de sensores e atuadores para monitorar e melhorar as condições físicas e mentais dos pacientes [32].

Contexto é definido por Dey (2001) como "qualquer informação que caracteriza a situação de uma entidade, sendo que uma entidade pode ser uma pessoa, um lugar ou um objeto, considerados relevantes para a interação entre um usuário e uma aplicação, incluindo o próprio usuário e a aplicação. O contexto é tipicamente a localização, a identidade e o estado das pessoas, grupos ou objetos físicos e computacionais". Para um sistema ser considerado ciente de contexto, ele deve fazer uso do contexto para fornecer informações ou serviços relevantes para o usuário, sendo que a relevância depende da tarefa executada pelo usuário $[6,30]$.

Os termos ciência de contexto e ciência de situação são usados por alguns autores como sinônimos. Para evitar essa confusão, é importante que nos foquemos no conceito de ciência de situação que se concentra na modelagem do ambiente do usuário. A ciência de contexto permite que os sistemas se adaptem automaticamente às mudanças no domínio de tarefas de um usuário, por meio da atualização de informações relevantes e de 
prestações de serviços, ao passo que ciência de situação tem foco em informações sobre o estado do ambiente onde essas tarefas são realizadas.

Endsley (1995) trata a Ciência de Situação como "a percepção e compreensão de uma ou mais situações e a projeção de seus efeitos em um futuro próximo" [12]. O modelo é formado por três níveis voltados para a obtenção de ciência de situação: percepção, compreensão e projeção. Em uma definição mais atual, trata-se situação como abstrações semânticas obtidas a partir de contextos de baixo-nível, permitindo especificações de alto-nível do comportamento humano no cenário e nos sistemas de serviços correspondentes. Ainda, define-se uma situação como uma visão de alto nível e abrangente de contexto, utilizada pelas aplicações em seu processo de adaptação.

Essa visão decorre da construção de contextos de alta complexidade, que podem ser criados a partir de informações obtidas por diferentes sensores distribuídos no ambiente ubíquo. Finalmente, afirma que a construção do suporte à ciência de situação para as aplicações ubíquas apresenta diversos desafios de pesquisa, tais como: a coleta do contexto a partir de fontes heterogêneas e distribuídas, o processamento das informações de contexto adquiridas e a respectiva atuação sobre o meio físico e a disseminação do contexto a consumidores interessados de forma distribuída e no momento oportuno.

\subsection{Saúde Ubíqua}

Saúde Ubíqua pode ser definida como a oferta de assistência médica em qualquer lugar, a qualquer momento, por meio de tecnologias de comunicação de banda larga e sem-fio móveis [33]. A saúde ubíqua também pode ser entendida como a utilização da infraestrutura de computação ubíqua como ferramenta de assistência à saúde [26, 30]. Ainda, através de um smartphone, dados de saúde de um paciente podem ser fácil e continuamente monitorados através de sensores embarcados de baixa potência para descrever o seu status e sintomas [34].

Saúda ubíqua é um campo emergente da tecnologia que usa uma grande variedade de sensores espalhados pelo ambiente e pelos pacientes com o objetivo de monitorar e melhorar a condição mental e física dos pacientes [35]. Pequenos sensores que coletam informações do corpo como temperatura, frequência cardíaca, pressão sanguínea e quase toda e qualquer característica fisiológica que fornecem informações que auxiliam em diagnósticos de saúde [36].

O propósito principal destes sensores é auxiliar pacientes e seus cuidadores de modo a analisar e realizar intervenções que melhoram o status de saúde. Com o tempo, a tecnologia se estenderá a todos os indivíduos, não somente aqueles com condições crônicas ou similares. Pacientes menos capazes, como crianças pequenas ou pessoas com problemas cognitivos precisam de suporte mais intensivo de profissionais e membros da família. Tecnologias de saúde ubíqua podem monitorar e alertar fatores que influenciam na saúde dos pacientes que englobam não somente a saúde física, como mental [37].

Tecnologias também estão sendo desenvolvidas para apoiar atividades de cuidadores, seja no hospital ou em outros ambientes críticos, assim como cuidados básicos. Exemplos incluem: sistemas EHR que modificam a informação mostrada aos funcionários do hospital baseada no contexto atual destes funcionários [38]; suporte à informação qualificada entre enfermeiras em troca de turno [39] e a pré-transmissão de informações de cenas de acidentes para hospitais [40].

\section{Modelo Proposto}

Esta seção apresenta o modelo PEPContextual, com ênfase nos componentes da arquitetura e na descrição dos artefatos integrantes do sistema em geral. Inicialmente, será apresentada a arquitetura do modelo PEPContextual, detalhando os componentes das camadas cliente e do serviço oferecido, agentes e premissas do projeto. Na sequência, será apresentada a estrutura da ontologia baseada no OpenEHR, utilizada na geração de indicadores de riscos do paciente através de dados de EHR. Finalmente, o foco é no detalhamento do estudo de caso utilizado para demonstrar as potenciais situações e a aplicabilidade do modelo. 


\subsection{Arquitetura}

A arquitetura do PEPContextual foi criada com o propósito de disponibilizar uma estrutura de software - no formato de um framework - e serviços para ambientes de computação e saúde ubíqua no qual a inferência de riscos fosse simplificada e adequada a um atendimento profissional e caracterizado pela mobilidade. A abordagem de apresentação do modelo é top-down, onde os módulos gerais da arquitetura são revelados inicialmente e os detalhes são mencionados em sequência.

A inferência de riscos proposta pelo modelo leva em consideração diversas formas de contexto, como informações do profissional, paciente e dados de prontuário eletrônico. No entanto, flexibiliza e incentiva a entrada de outras formas e sensores, como localização, RFID, entre outros. Para representação deste conhecimento foram escolhidos fragmentos de ontologias baseadas no padrão OpenEHR, vista como essencial para o modelo realizar a inferência de riscos e permitir a interoperabilidade e integrabilidade. Estas informações do paciente compreendem indicadores de sintomas, histórico médico e ambiente no qual ele se encontra.

A Figura 1 revela em alto nível o fluxo de ação e aplicação do modelo. Primeiramente são gerados os dados no sistema de origem e exportados para um arquivo e importados para uma base de dados relacional (passo 1). Então um Gateway - serviço que ficará executando no servidor, denominado Sistema de Coleta de Prontuário - irá consumir estes dados e convertê-los no modelo da ontologia baseada no OpenEHR (passo 2). A partir desse momento, existem duas formas da ativação das inferências: através da ação do profissional pelo aplicativo ou pelo próprio gateway, disparando o motor de inferência, que analisa os dados da ontologia e avalia se há alguma inferência a ser realizada (passo 3). Caso exista, uma ação correspondente a essa inferência é disparada (passo 4). Caso contrário, apenas espera-se uma nova geração de dados no sistema de origem, um novo ciclo do gateway ou uma ação do profissional através do aplicativo (passo 5).

Figura 1: Fluxo de Ação e Aplicação do Modelo.

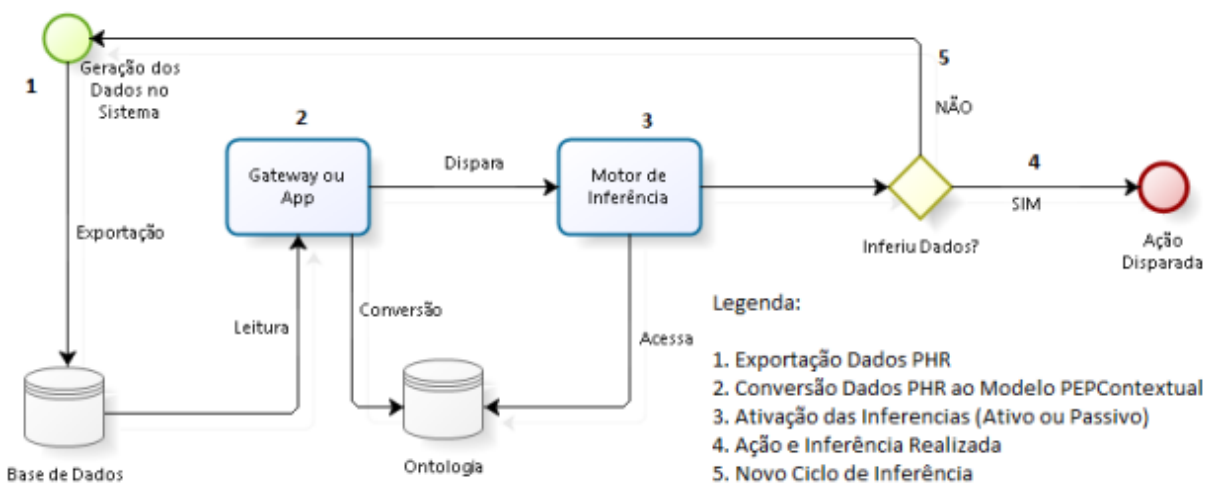

O contexto extraído do dispositivo é enviado para o servidor através de um web service REST. Essas informações contextuais, juntamente ao gateway de coleta de dados - representado através do Sistema de Coleta de Prontuário - abastecem a ontologia baseada no OpenEHR e o Motor de Inferência que irá determinar, através de regras - fazendo uso de consultas LINQ - se há algum sintoma ou enfermidade constatados e, se necessário, alguma ação a ser disparada.

O modelo da arquitetura exibe diferentes módulos que se comunicam com o intuito de efetuar a captação e análise de contexto e entregando inferências. Para tanto, os dados do profissional de saúde, do paciente e informações do prontuário eletrônico devem estar armazenados nos arquétipos da ontologia baseada no OpenEHR. Na arquitetura proposta, esse armazenamento, captação e controle dos dados é realizada pelo Repositório de Ontologias. Esses dados são fundamentais na busca por inferências de sintomas ou diagnósticos, tendo em vista o grau de incerteza que existe durante um atendimento hospitalar. Após a detecção da necessidade de entrega de informações através de inferências, representado pelo Motor de Inferência, o servidor emite uma notificação através do Sistema de Notificação, responsável por enviar para o dispositivo do profissional um alerta em caso de uma inferência pertinente ao tratamento. O serviço de notificações segue o fluxo em apenas uma direção, sendo responsável por enviar a mensagem ao aplicativo instalado. Com o recebimento da notificação, o profissional acessa em seu dispositivo o aplicativo cliente - que coleta o contexto do próprio profissional, como por exemplo especialidade - e visualiza as inferências realizadas que podem auxiliar no tratamento. 
No serviço estão contidos sistemas de contexto, risco, notificação e coletor, representados respectivamente pelos agentes de contexto, risco e notificação, bem como o agente coletor. O agente coletor é encarregado de ler a base de dados e normalizar as informações na ontologia baseada no OpenEHR. O agente de contexto é responsável por avaliar o contexto de uma informação e/ou entidade. O agente de risco é o motor de inferência que irá analisar os dados contidos na ontologia, bem como o contexto envolvido e acionar o agente de notificação. Ainda, há a interface REST que expõe os comportamentos dos agentes que compõem os sistemas. A aplicação cliente se encarrega de coletar o contexto do profissional utilizador, bem como servir de interface de informações de um paciente e receber as notificações de inferência realizadas pela aplicação servidor.

\subsection{Serviço REST}

O serviço do PEPContextual deve conhecer o contexto do profissional, do paciente e os prontuários eletrônicos vinculados ao paciente. Com posse dessas informações de contexto e dados históricos, é iniciado o processo de inferência com a utilização do método LINQ (Language Integrated Query) e aplicação das regras de inferência definidas. A ontologia é responsável por representar o conhecimento e padronizar a estrutura de dados médicos.

A aplicação divide-se em componentes para que possa prover as informações necessárias aos processos. A metodologia utilizada para a modelagem dos agentes é a Prometheus, pois define um processo em detalhes para a etapa do desenvolvimento do sistema orientado a agentes. Além disso, como o modelo PEPContextual executa monitoramento e inferências continuamente de modo autônomo, o uso de agentes e da metodologia citada foram escolhidos. Basicamente, 4 agentes compõem a estrutura de inferência que corresponde a camada servidor:

- O agente coletor, responsável por ler a base de dados e normalizar os dados na ontologia OpenEHR. Este agente insere marcações de contextos sempre um ciclo do gateway de coleta encontra informações disponíveis. Ainda, ele faz o de-para das informações conforme o arquétipo utilizado;

- O agente de contexto, responsável por avaliar o contexto de uma informação ou entidade. Nesse sentido, o agente de contexto prepara as informações para que o agente de risco possa realizar inferências de sintomas ou indicativos de diagnósticos;

- $\quad$ O agente de risco, encarregado de analisar os contextos filtrados pelo agente de contexto e aplicar as inferências de acordo com o contexto escolhido, avaliando se as inferências estão relacionadas com algum sintoma ou diagnóstico indicado. Este agente aciona o agente de evento em caso de uma inferência positiva. Ainda, guarda o histórico de inferências para auxiliar em resultados futuros;

- $\mathrm{O}$ agente de notificação, responsável por enviar status das inferências deste usuário sempre que acontecem.

\subsection{Cliente}

A arquitetura proposta para a camada cliente é composta pelos módulos de coleta de contexto e interface gráfica. O contexto dinâmico, como a localização ou características do dispositivo é capturado no momento que o profissional realiza o Login do sistema.

Ao realizar a busca por informações do prontuário eletrônico de um paciente específico, o Módulo de Inferência dispara o motor de inferência com os dados da ontologia. A notificação enviada ao profissional é um recurso necessário para alertá-lo que existe uma ou mais inferências novas, e que esses resultados são relevantes ao diagnóstico ou tratamento. Esse recurso de notificações está presente nos smartphones mais utilizados, como é o caso do Android. Com a ajuda desta característica no aplicativo cliente é importante saber como processar essas informações (encapsulamento de notificações) e como exibi-las para o profissional. Esse processamento é realizado pelo Sistema de Notificação.

\subsection{Cenário de Aplicações}

Foi pensado um cenário específico de aplicação do modelo com foco em saúde mental, onde um profissional de saúde, aplica dois questionários em um paciente e espera uma ou mais inferências relacionadas. 
A seguir são exibidos os dois questionários de acompanhamento realizados pelos profissionais de saúde mental que disparam inferências e análises de risco automáticas.

\subsubsection{Rastreamento SRQ (Self-Reporting Questionnaire)}

Um dos métodos de baixo custo para rastreamento psiquiátrico é o SRQ (Self-Reporting Questionnaire), instrumento de rastreamento de transtorno mental. O SRQ é composto originalmente por 30 questões, sendo 20 sobre sintomas psicossomáticos para rastreamento de transtornos não-psicóticos, quatro para rastreamento de transtornos psicóticos, uma para rastreamento de convulsões do tipo tônico-clônica e cinco questões para rastreamento de transtorno por uso de álcool. Além disso, está recomendado pela OMS para estudos comunitários e em atenção básica à saúde, principalmente nos países em desenvolvimento, por preencher os critérios citados acima em termos de facilidade de uso e custo reduzido [42].

\subsubsection{Piloto do Hospital Mãe de Deus - ARC (Avaliação de Risco do Comportamento)}

A mudança de "risco psiquiátrico" para "risco do comportamento" significa que as pessoas em risco podem ter ou não um transtorno mental. Além disso, existem várias condições que podem influenciar o comportamento e são comuns em hospitais (por exemplo: alterações metabólicas, infecções, efeitos de medicamentos). Finalmente, ter um transtorno mental como depressão ou psicose, geralmente aumenta o risco, mas no momento da avaliação inicial o risco pode não estar presente [43].

O protocolo ARC revela uma avaliação através da verificação 7 itens que compõe o modelo, as quais devem ser assinaladas "sim" caso esteja presente algum dos itens, ou "não" em caso de ausência; a presença de "sim" em um ou mais dos itens já significa que há risco, sem que isso gere um escore. Ainda, a equipe de saúde mental (Psiquiatria, Psicologia, Serviço Social, Terapia Ocupacional) será acionada de acordo com cada caso, após ser comunicado o médico assistente [43].

\subsection{Comportamento do Modelo}

A técnica de ciência de situação envolve praticamente todos os componentes do modelo. Baseado no modelo de Endsley, ela utiliza três níveis para conseguir detectar as situações de risco. No primeiro nível são coletados os dados relevantes que são as informações de perfil do usuário (contexto de perfil) e as informações do paciente. No segundo nível é feito o processamento dos dados coletados e trata das inferências, permitidas pelos arquétipos no domínio de PHR e das informações de contextos de perfil. E finalmente no último nível, é feita a inferência dos riscos associados. A seguir, são detalhados cada um dos passos envolvidos no processo:

- Passo 1 - Envio dos dados de autenticação do usuário e o nome do profissional para o servidor de aplicação;

- Passo 2 - A partir da validação das credenciais do usuário, informadas no passo 1 , o servidor de aplicação consulta na base de dados de perfil os pacientes deste profissional;

- Passo 3 - Envio de informações ao serviço de inferência o nome do paciente e suas informações do PHR;

- Passo 4 - O serviço de consulta a base de conhecimento em busca protocolos que contenham informações, inferindo riscos associados;

- Passo 5 - O serviço de inferência informa ao servidor de aplicação os dados retornados da consulta feita no passo 5;

- Passo 6 - Por último, caso o servidor de aplicação constate que foram retornados no passo 5 informações de riscos, apresentará para o profissional quais riscos associados e quais ações estão vinculadas a essa inferência. Caso o passo 5 não retorne nenhum valor, interferirá que não existem riscos potenciais ligados ao paciente. 
Mostrou-se que com a utilização dos contextos de perfil e de dados de PHR foi possível realizar as inferências pretendidas. O contexto de perfil permitiu que o modelo identificasse o contexto do profissional. Ainda, a correlação entre profissional e PHR permite identificar se existem riscos à saúde do paciente. Essa correlação só é possível graças aos arquétipos utilizados, que armazenam todas as informações sobre os dados de PHR utilizados para a inferência de riscos associados.

\section{Implementação}

Nesta seção são apresentados os aspectos de implementação do modelo, bem como do serviço e da aplicação cliente. Ainda, revela as avaliações pelas quais este trabalho será submetido juntamente com os resultados esperados. Para isso, este capítulo será dividido de forma a exibir as metodologias utilizadas para a avaliação do modelo, bem como a avaliação do protótipo do modelo PEPContextual.

Para o desenvolvimento do modelo de inferência de riscos, do aplicativo baseado no modelo proposto e visando levantar e qualificar os requisitos necessários, foi desenvolvido um fluxograma de exemplo de atividades do modelo, representado pela Figura 2. Nela, o usuário efetua o Login no aplicativo, acessando o serviço e realiza uma busca de paciente e seus dados de PHR. Ainda, ativa uma ação de inferência (explícita) e recebe um feedback visual ou via notificação. Feito isso, com base nestes mecanismos foi criado um diagrama de componentes de alto nível, representado pela Figura 3, com o intuito de avaliar a quantidade de entidades a serem utilizadas, bem como foram definidas as ferramentas de trabalho, ambiente, metodologias, padrões e arquitetura de desenvolvimento, organização das camadas, etc. Os componentes descritos são:

Figura 2: Diagrama de Atividades.

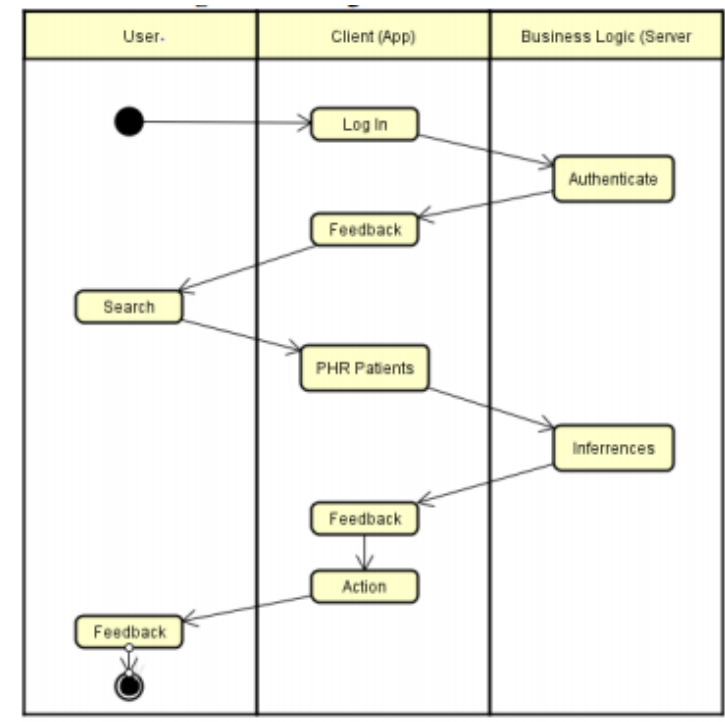

- Aplicação Cliente, com a interface do usuário e as ações backoffice - como inferências via pooling ou coleta de contexto para formar a situação;

- Aplicação Servidor, com as interfaces de $\mathrm{E} \backslash \mathrm{S}$ e os agentes de risco, contexto, notificação e coletor;

- Base de Dados, onde o modelo dos arquétipos é armazenado em JSON, bem como o modelo de logs para análise e checagem das inferências realizadas;

- $\quad$ Barramento, onde ficam expostas as camadas de integração.

A plataforma escolhida para implementação do aplicativo foi o Android por ser a plataforma móvel mais utilizada e, portanto, passível de atingir o número de usuários em um curto espaço de tempo. A arquitetura envolve, além do aplicativo citado, serviços expostos com os procedimentos utilizados nos processos, uma ontologia para armazenar as informações prontuário eletrônico e agentes, cada um com sua responsabilidade, 
além de um serviço de notificações. Esse recurso de notificações está presente nos smartphones mais utilizados como é o caso do Android - plataforma escolhida para o desenvolvimento do protótipo. Além de ser desenvolvido em Linux, é livre, que já é de fato uma característica importante, possui uma interface visual rica em detalhes e várias aplicações que vem pré-instaladas que proporcionam para o desenvolvedor um ambiente favorável.

Figura 3: Diagrama de Componentes.

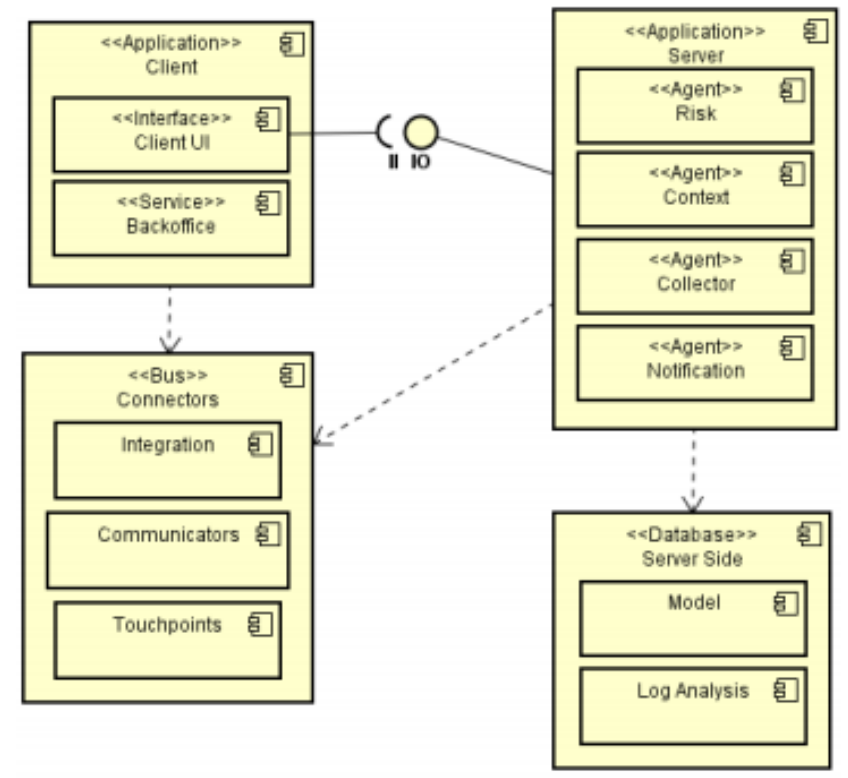

A arquitetura completa da solução, envolve, além do aplicativo para Android, um servidor de aplicações e um banco de dados para armazenar as informações dos estabelecimentos e disponibilizá-las para o aplicativo. Para desenvolvimento do aplicativo, foi utilizada a IDE Android Studio. Para desenvolver o servidor de aplicações, foi utilizado o .NET Framework e a IDE Visual Studio, além de um serviço criado utilizando a tecnologia WCF (Windows Communication Foundation), expondo um REST.

Portanto, destacam-se três grandes nodos na arquitetura: o aplicativo Android, o serviço e o banco de dados. No nodo servidor, a aplicação ainda se divide em diversos componentes para que o mesmo possa prover as informações necessárias ao aplicativo. Neste nodo, a arquitetura está dividida em 6 grandes componentes. Destacam-se as camadas de regra de negócio e de acesso a dados, que estão devidamente separadas na arquitetura do software a fim de fornecer maior manutelibilidade.

\subsection{Serviço REST}

De um modo geral, a camada cliente baseia-se numa aplicação criada para Android. No lado servidor e através da interface REST, camadas internas com diferentes responsabilidades com o objetivo de ter uma estrutura robusta, que diminua o tempo de desenvolvimento e proporcione uma baixa manutelibilidade. Ainda, o conceito de arquitetura pode ser expandido para outras plataformas, como websites ou outros dispositivos ubíquos de diferentes plataformas, porém sempre preservando a arquitetura previamente definida.

O serviço foi criado utilizando a tecnologia WCF (Windows Communication Foundation), expondo um REST. A comunicação do aplicativo com o serviço ocorre através do protocolo HTTP (Hypertext Transfer Protocol), utilizando a função GET. Ou seja, o serviço recebe as chamadas e retorna dados no formato JSON (JavaScript Object Notation) - formato de troca de informações amplamente utilizados em aplicações para dispositivos móveis, além de possuir performance para transmissão dos dados.

A camada de acesso a dados utiliza a ontologia para realizar consultas e persistências. Foram criadas as camadas de serviço, a camada de DTO's (Data Transfer Object) e a camada de conversão de dados, o Parser. A camada de serviço fornece os métodos que serão utilizados pelos processos e a camada Parser é responsável por 
converter as informações da camada de modelo para objetos de classes menos complexas e mais acessíveis para serem transmitidas através do protocolo HTTP utilizando o formato JSON para o aplicativo.

Como um exemplo de comportamento do serviço, o usuário acessa o aplicativo e realiza seu Login. $\mathrm{O}$ aplicativo acessa o serviço, que recebe um DTO, convertido pelo Parser em um Model. A camada BL (Business Logic) aplica as regras e faz uso da camada DA (Data Acess) para retornar as informações salvas no formato JSON salvas no BD (Database). Retornados os dados e aplicado o devido tratamento, as informações no formato Model são novamente convertidas em DTO pelo Parser e expostas ao aplicativo.

\subsection{Aplicativo Cliente}

A arquitetura proposta para a camada cliente é composta por módulos macro: coleta de contexto, agente de análise de riscos e interface gráfica. Na arquitetura do aplicativo também foi utilizada a camada de DTO, a camada Parser e uma camada de serviço. Além disto, a arquitetura prevê a utilização da camada de visualização, contendo as interfaces de usuário do aplicativo e sua lógica de interação, além de uma camada Adapter, que é a camada específica para renderização e tratamento das listas de informações do aplicativo.

A camada de interface comunica-se com os threads para simular as chamadas assíncronas para a camada de serviço que, por sua vez, realiza a chamada HTTP para o serviço no servidor de aplicações. Após obter o retorno, o JSON de resposta é convertido pela camada Parser em objetos DTO, que são a representação reduzida das entidades de negócio que devolve para a camada as informações em já convertidas para a camada de interface. A camada de interface pode exibir as informações diretamente na tela, passar as informações para a camada Adapter para exibição das informações em uma lista ou mesmo executar qualquer outra ação pertinente ao contexto.

Como um exemplo de comportamento do cliente, o usuário acessa o aplicativo e realiza seu Login. O aplicativo acessa primeiramente uma interface de serviço no cliente, que faz uma chamada REST ao o serviço no lado servidor, enviando um DTO, convertido pelo Parser a partir de um Model. A camada serviço REST retorna as informações devidamente tratadas no formato DTO, onde são convertidas em BO (Base Objects) pelo Parser e disponibilizadas na tela do aplicativo pelo Adapter, que realiza as transformações e adaptações dos dados ao formato de visualização.

\section{Avaliação do Modelo}

Este capítulo possui os principais aspectos abordados na avaliação do modelo, bem como metodologia, questionário aplicado, resultados, facilidade de uso, utilidade e opiniões dos usuários.

\subsection{Metodololgia}

Nesta seção são apresentadas as avaliações pelas quais este trabalho foi submetido juntamente com os resultados esperados. Salienta-se que todos os dados fornecidos foram reais, sejam os de pacientes pesquisados e avaliados, quanto dos profissionais utilizadores.

Para isso, a avaliação do PEPContextual consistiu na utilização do protótipo para execução de dois tipos de avaliação. O primeiro tipo de avaliação consistiu no uso estendido do protótipo com duração de 14 dias, através de um estudo de caso. A segunda avaliação consiste na comparação das inferências obtidas pelo modelo PEPContextual versus as inferências esperadas.

\subsection{Avaliação de Uso Estendido do PEPContextual}

Esta avaliação teve duração de 14 dias e foi realizada pela equipe de equipe de saúde mental (Psiquiatria, Psicologia, Serviço Social, Terapia Ocupacional) do Hospital Mãe de Deus de Porto Alegre. A avaliação foi aplicada com o objetivo de coletar dados de contexto de pacientes, profissionais utilizadores e realizar as inferências que pudessem vir a auxiliar em diagnósticos e/ou sintomas.

Após esse período, foi solicitado que os participantes respondessem, um questionário que avaliou o impacto do modelo em casos reais nos quais este fora aplicado. O objetivo deste questionário foi validar a percepção do profissional sobre a aplicabilidade do modelo em um ambiente real.

Revista Brasileira de Computação Aplicada (ISSN 2176-6649), Passo Fundo, v. 9, n. 2, p. 43-58, jul. 201752 
O questionário principal tem caráter quantitativo, com algumas questões qualitativas, relativas a opinião dos utilizadores em relação ao PEPContextual. As opções de respostas para as perguntas quantitativas seguiram o padrão da escala de Likert de cinco pontos, variando entre 1 (discordo totalmente) até 5 (concordo totalmente) [44].

As perguntas foram elaboradas com base nos conceitos do o questionário para análise de satisfação do modelo proposto foi construído utilizando a metodologia TAM (Tecnology Acceptance Model). Ao total, para os testes foram utilizados 5 usuários distintos da equipe de saúde mental do Hospital Mãe de Deus de Porto Alegre atualmente o aplicativo conta com 12 profissionais ativos. O modelo TAM considera os seguintes aspectos como as influências determinantes para a aderência de uma nova tecnologia:

- Facilidade de Uso: percepção na qual uma pessoa acredita que a tecnologia influencia e facilita seus esforços;

- Utilidade: percepção na qual uma pessoa acredita que a tecnologia melhora o desempenho das atividades.

\subsection{Resultados do Questionário de Usuário}

Os perfis dos usuários que exploraram o modelo e aplicativo correspondem, conforme os resultados, observa-se que a maior concentração de profissionais se encontra na faixa "Entre 20 e 30 anos", com 60\%. Quanto ao sexo, temos uma maioria pertencente ao "Masculino", com os mesmos 60\%.

Todos os profissionais têm alguma graduação, com 40\% com pós-graduação, O que faz total sentido, visto que todos os profissionais são médicos formados. Ainda, foram feitas 3 perguntas: A primeira era se "Você costuma utilizar smartphones ou tablets?", a qual foi respondia com "Sim" por 100\% dos profissionais. A segunda fora a respeito do tempo de utilização semanal, onde $80 \%$ responderam que utilizam o smartphone por mais de 15 horas. Apenas um profissional declarou utilizar "Entre 3 e 5 horas", o que parece estar ligado ao seu perfil de idade. Finalmente, a terceira era sobre qual plataforma de smartphones os profissionais estavam habituados a utilizar, com $60 \%$ declarando ter preferência pelo iOS e o restante dos $40 \%$ pelo Android. Este dado sugere que uma versão do aplicativo para o iOS teria uma aceitação pela maioria dos utilizadores.

Entrando nas questões de facilidade de uso, conforme revela a Figura 4, 100\% dos utilizadores concordou totalmente que o aplicativo é de fácil utilização e $80 \%$ concordaram totalmente que a interface revela boa visualização e entendimento das informações com pouco esforço. Ainda, $60 \backslash \%$ concordaram totalmente que a pesquisa e visualização de um paciente é intuitiva, enquanto $40 \%$ concordaram parcialmente. Este indicador revela a necessidade de um esforço na inclusão de novas funcionalidades que alterem a percepção do profissional de forma positiva.

Figura 4: Facilidades de Uso percebida pela utilização do PEPContextual.

Com pouco esforço, visualizo e entendo as informações e campos dispostos É possivel pesquisar um paciente, bem como visualizar suas informaçōes e nas interfaces. protocolos de forma intuitiva.
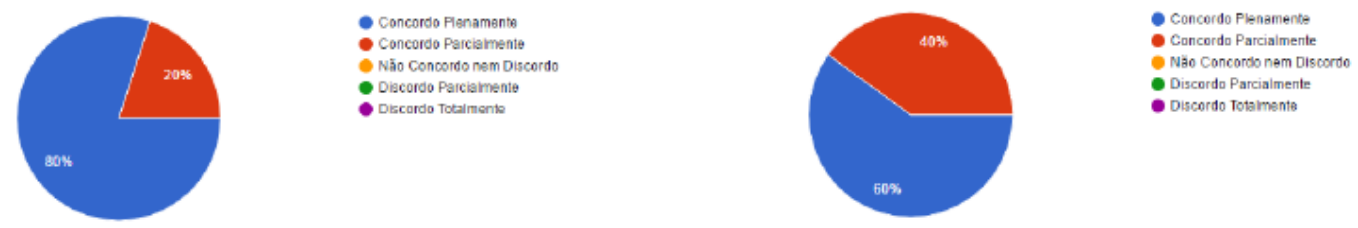

Tanto o fato da interface disponibilizar todas as informações que o aplicativo necessita para sua utilização quanto o de o aplicativo deixar claro o que está sendo avaliado ou inferido, $60 \%$ dos profissionais concordaram totalmente, com os $40 \%$ restantes concordando parcialmente. Este aspecto indica que informações podem estar fazendo falta para o profissional e que as mensagens das inferências podem ser melhor trabalhadas.

Entrando nas questões de utilidade do sistema, conforme mostra a figura 5, tanto o fato do aplicativo deixar clara sua contribuição quanto o fato de o aplicativo revelar utilidade e aderência aos processos hospitalares, $60 \%$ dos profissionais concordaram parcialmente, com os $40 \%$ restantes concordando totalmente. 
Este aspecto indica que existem aspectos de interface ou disposição de informações a respeito do aplicativo que devem ser melhor desenvolvidas em versões posteriores.

Figura 5: Utilidade do Sistema PEPContextual percebida pelos usuários com relação à contribuição e utilização.

O aplicativo deixa claro qual o seu propósito e contribuição. (5 respostas)

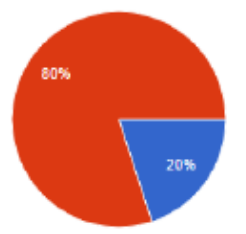

- Concordo Planamenta

Considerando a utilização, bem como inferências realizadas e informações do pacientes visualizadas, pode-se afirmar que o aplicativo revela utilidade e aderência aos processos hospitalares.

(5 respostas)

Destaca-se, conforme a Figura 6 , que $60 \%$ dos profissionais concordaram parcialmente que os protocolos demonstrados (SRQ e ARC) são um bom ponto de partida para revelar as possibilidades de inferências pelo modelo, com os $40 \%$ restantes concordando totalmente. Ainda, os mesmos $60 \%$ revelaram concordar parcialmente a respeito das mensagens e notificações e sua utilidade em inferências sobre os protocolos aplicados. Tivemos $20 \%$ de profissionais neutros - "Não Concordo nem Discordo" - e 20\% concordando totalmente. Novamente, destaca-se a necessidade de clareza nas afirmações e informações inferidas, visto a natureza e origem dos dados.

Figura 6: Utilidade do Sistema percebida pelos usuários do PEPContextual com relação as inferências realizadas

Os protocolos demonstrados no aplicativo são um bom ponto de partida para As notificaçŏes e mensagens do aplicativo demonstram utilidade, além de revelar as possiblidades de realização de outras inferências, dentro ou fora da área de saúde mental. revelar inferências sobre os protocolos aplicados. da área de
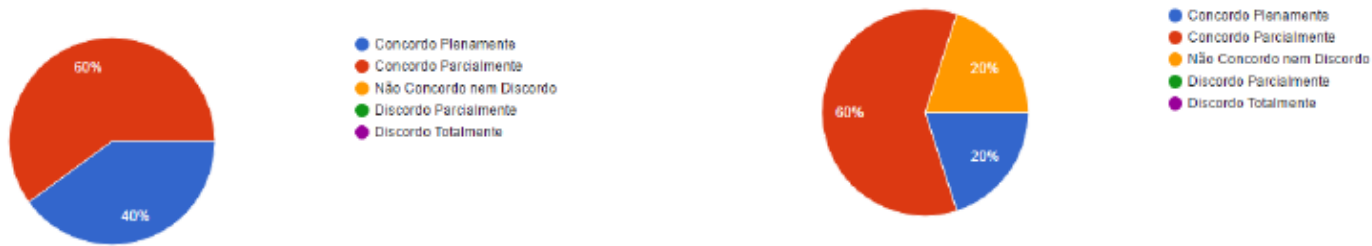

Ainda, $60 \%$ dos profissionais concordam totalmente quanto ao fato das informações utilizadas e inferidas no aplicativo serem importantes para um acolhimento e triagem da saúde mental. Quanto ao aspecto do aplicativo se aplicar a outros cenários de maneira suficiente, $60 \%$ dos profissionais concordaram totalmente e os $40 \%$ restantes concordaram parcialmente. No que tange a questão do auxílio das informações inferidas diariamente em tratamentos, A Figura 7 revela que $80 \%$ dos profissionais concordaram parcialmente e $20 \%$ concordaram totalmente. Quanto a afirmação de que as informações inferidas poderiam auxiliar diretamente em diagnósticos, a maioria $(60 \%)$ concordou totalmente, com $20 \%$ se dividindo entre neutro e os que concordaram parcialmente.

Figura 7: Utilidade do Sistema PEPContextual considerando o uso das informações pelos usuários. diagnósticos.

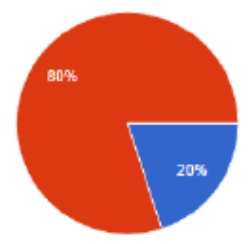

- Concordo Plenamente - Concordo Pardialnents - Náo Concordo nem Discor do Discordo Toramerie

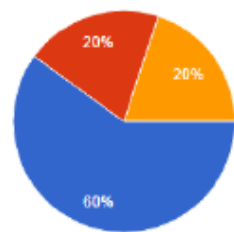

Conourdo Plenamente Naco corcosiamente Discordo Pardam biscerdo - Discordo Totaimente

Finalmente, tratando-se da relevância das opções de funcionalidades do aplicativo, $60 \%$ dos profissionais concordaram parcialmente e $40 \%$ totalmente. Quanto ao aplicativo estimular a troca de informações dos pacientes entre os profissionais, a Figura 8 revela que $80 \%$ concordaram parcialmente e $20 \%$ se 
manteve neutro. Destaca-se que não houve nenhuma resposta negativa ("Discordo Totalmente" e "Discordo Parcialmente") durante a avaliação. No que tange as questões qualitativas, os profissionais indicaram a sensibilidade do modelo e seu potencial aplicado ao protótipo.

Figura 8: Utilidade do Sistema PEPContextual quanto a relevância das informações e incentivo a comunicação.
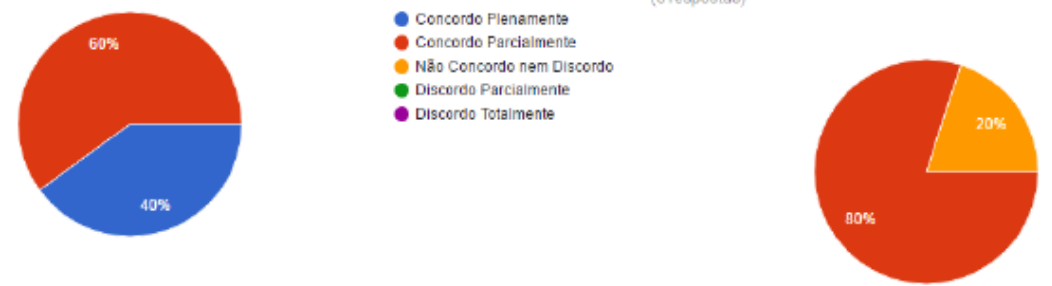

- Concordo Plenamenta - Concordo Pardialmente Discrerdo Parciamente Discordo Totalmente

Citaram as possibilidades de prever riscos, realizar triagem e antecipar medidas em tratamentos ou diagnósticos. Sobre os pontos negativos, destaca-se o fato de não haver uma sinalização nos pacientes já avaliados, como uma espécie de status e a falta de possibilidade de inserir informações de prontuário e evolução do quadro. Sugeriu-se ainda a possibilidade de ordenar a lista de pacientes por ordem alfabética ou "favoritar" o mesmo e, um ponto importante, a necessidade de integrar o modelo do hospital com os dados do aplicativo. Atualmente é realizada uma carga diária e os potenciais de acesso e visualização com o acesso direto ao modelo de dados seriam enormes.

Em se tratando de pontos positivos, ressaltou-se a facilidade no manuseio e na natureza intuitiva do aplicativo. Quanto aos principais benefícios do uso do aplicativo no auxílio de um tratamento ou diagnóstico, destacou-se o fato de favorecer a comunicação entre a equipe e sinalizar para a mesma quais os passos do tratamento que necessitam de maior atenção.

\section{Considerações Finais}

Este trabalho apresentou um novo modelo para a inferência de riscos de pacientes através de dados PHR fazendo uso da ciência da situação. Além disso, foi feito um levantamento detalhado sobre os frameworks e modelos disponíveis no mercado. Nesse estudo, foi possível identificar as principais características de cada um, aplicações e tecnologias utilizadas. O modelo PEPContextual reuniu as principais características positivas entre os trabalhos relacionados e protótipo do aplicativo foi desenvolvido atendendo as funcionalidades fundamentais propostas pelo modelo.

O modelo consistiu em um PHR focado para dispositivos moveis, no âmbito da saúde ubíqua que utiliza a ciência da situação a fim de gerar informações de risco ao paciente de acordo com o contexto do usuário. Assim sendo, o presente trabalho apresentou um modelo ubíquo baseado em ciência de situação para detecção de riscos associados ao paciente, conforme contextos do usuário. O modelo propôs o suporte de dispositivos móveis, com a aplicação de computação ubíqua e da utilização de ciência de situação. Após a escolha do tema, estudou-se os conceitos de computação ubíqua, contexto, ciência de situação, entre outros. Esse estudo serviu de embasamento e colaborou na análise crítica dos trabalhos relacionados.

O protótipo do modelo foi projetado utilizando tecnologias abertas, com cliente representado por um aplicativo nativo em Android. No lado servidor desenvolveu-se o servidor de aplicação em .NET executado no IIS (Internet Information Services), com a base de dados sendo armazenada no banco de dados SQLServer. O serviço de inferência utilizou consultas LINQs e regras pré-definidas. O servidor de aplicação está armazenado na plataforma IIS juntamente a base de dados Microsoft SQL Server, ambos na Plataforma AWS (Amazon Web Services) em uma instância t2.micro com 1GB de memória RAM e 30 GB de storage EBS.

O modelo foi avaliado das seguintes maneiras: a primeira avaliação foi feita através de um estudo de caso e a segunda avaliação contemplou a usabilidade do aplicativo. A avaliação por estudo de caso confirmou a expectativa de que a aplicação de ciência de situação, baseada no modelo de Endsley, possibilitaria que o modelo de forma ubíqua detectasse riscos associados ao paciente. Ainda, as perguntas foram aplicadas quando o uso do 
aplicativo fora finalizado, bem como também foram aceitas melhorias e críticas. Visou-se buscar perspectivas para novas aplicações do modelo, dentro ou fora do âmbito da saúde mental.

O modelo PEPContextual buscou uma arquitetura flexível, de modo a aumentar sua aderência e adaptação a outros modelos e sistemas. No processo de inferência, muitos dos trabalhos têm técnicas próprias, como Redes Bayesianas e algoritmos específicos. O modelo PEPContextual fez uso de consultas LINQs e regras pré-definidas, porém a flexibilidade na escolha das técnicas utilizadas foi uma das características propostas.

Qualquer dado que um paciente gera em um tratamento de saúde é relevante e importante. Diversos sistemas cientes de contexto fazem uso de estruturas de dados específicas para representar contexto e acabam por apresentar soluções restritas, não flexíveis ou parcialmente integráveis a outros modelos. A utilização de arquétipos de referência na área da saúde por parte do trabalho proposto visou mitigar essas dificuldades. Além disso, nenhum dos trabalhos considerados representou uma solução completa para a computação ou saúde ubíquas, levando-se em consideração todos os desafios presentes da área.

Pode-se concluir com o trabalho que os objetivos foram alcançados de acordo com o que fora proposto inicialmente. Os aspectos abordados pelo trabalho são um bom indício para seguir trabalhando e novas pesquisas, alterações e ampliações do modelo PEPContextual são não somente possíveis, como estimuladas. Finalmente, entende-se que a aceitação do modelo foi clara e que trabalhos futuros poderiam agregar maior valor, relevância e conclusões para o tema e para a pesquisa.

\section{Agradecimentos}

Gostaríamos de agradecer a CAPES, FAPERGS e CNPq pelo apoio a esse projeto. Agradecemos também o Sistema de Saúde Mãe de Deus pela parceira no projeto e por disponibilizar sua equipe e infraestrutura para avaliação da pesquisa.

\section{Referências}

[1] DIAZ A.; MERINO, P. R. F. Mobile Application Profiling for Connected Mobile Devices. IEEE Pervasive Computing, [S.1.], v. 9, n. 1, p. 54-61, 2010.

[2] SATYANARAYANAN, M. Fundamental Challenges in Mobile Computing. In: FIFTEENTH ANNUAL ACM SYMPOSIUM ON PRINCIPLES OF DISTRIBUTED COMPUTING, 1996, New York, NY, USA. Proceedings. .. ACM, 1996. p. 1-7. (PODC '96).

[3] CACERES C.; FERNANDEZ, A. O. S. V. M. Agent-Based Semantic Serverice Discovery for Healthcare: an organizational approach. In: INTELLIGENT SYSTEMS, IEEE, 2006. Anais. . . [S.1.: s.n.], 2006. p. 1120.

[4] WEISER, M. In: BAECKER, R. M.; GRUDIN, J.; BUXTON, W. A. S.; GREENBERG, S. (Ed.). Humancomputer Interaction. San Francisco, CA, USA: Morgan Kaufmann Publishers Inc., 1995. p. 933-940.

[5] BELlaVista P.; CORRADI, A. F. M. F. L. A survey of context data distribution for mobile ubiquitous systems. ACM Computing Surveys, [S.1.], v. 44, n. 4, 2012.

[6] DEY, A. K. Understanding and Using Context. Personal Ubiquitous Comput., London, UK, UK, v. 5, n. 1, p. 4-7, Jan. 2001.

[7] CHEN, G.; KOTZ, D. A Survey of Context-Aware Mobile Computing Research. Hanover, NH, USA: Dartmouth College, 2000.

[8] HOFER, T.; SCHWINGER, W.; PICHLER, M.; LEONHARTSBERGER, G.; ALTMANN, J.; RETSCHITZEGGER, W. Context-Awareness on Mobile Devices - the Hydrogen Approach. In: ANNUAL HAWAII INTERNATIONAL CONFERENCE ON SYSTEM SCIENCES (HICSS'03) - TRACK 9 VOLUME 9, 36., 2003, Washington, DC, USA. Proceedings. . .IEEE Computer Society, 2003. p. 292.1-. (HICSS '03).

[9] SIMON S. K.; ANBANANDHEN, S. M. S. L. A Ubiquitous Personal Health Record (uPHR) Framework. In: ACM, 2013. Anais. . . [S.1.: s.n.], 2013.

Revista Brasileira de Computação Aplicada (ISSN 2176-6649), Passo Fundo, v. 9, n. 2, p. 43-58, jul. 201756 
[10] KHARRAZI, H.; CHISHOLM, R.; VANNASDALE, D.; THOMPSON, B. Mobile personal health records: an evaluation of features and functionality. International Journal of Medical Informatics, [S.1.], v. 81, n. 9, p. $579-593,2012$.

[11] SOUZA, M. V. B. Inferência de atividades clínicas na arquitetura clinicspace a partir de propriedades do contexto [dissertação]. In: UNIVERSIDADE FEDERAL DE SANTA MARIA, 2010. Anais. . . [S.1.: s.n.], 2010. p. 153 .

[12] ENDSLEY, M. R. A comparative analysis of SAGAT and SART for evaluations of situation awareness. In: IN PROCEEDINGS OF THE HUMAN FACTORS AND ERGONOMICS SOCIETY 42ND ANNUAL MEETING, 1998. Anais. . . [S.1.: s.n.], 1998. p. 82-86.

[13] DU, W.; WANG, L. Context-aware Application Programming for Mobile Devices. In: C3S2E CONFERENCE, 2008., 2008, New York, NY, USA. Proceedings. . . ACM, 2008. p. 215-227. (C3S2E '08).

[14] ANAGNOSTOPOULOS, C.; NTARLADIMAS, Y.; HADJIEFTHYMIADES, S. Situational computing: an innovative architecture with imprecise reasoning. Journal of Systems and Software, [S.1.], v. 80, n. 12, p. 1993 - 2014, 2007. Selected papers from the International Conference on Pervasive Services (ICPS 2006).

[15] STIPKOVIC, S.; BRUNS, R.; DUNKEL, J. Pervasive Computing by Mobile Complex Event Processing. In: ICEBE'13, 2013. Anais. . . [S.1.: s.n.], 2013. p. 318-323.

[16] COMMISSION, B. The state of broadband 2012: achieving digital inclusion for all. In: 2012. Anais. . . [S.1.: s.n.], 2012.

[17] MOBILE, C. Cisco Visual Networking Index: global mobile data traffic forecast update, 2011 to 2016. In: S.L.], 2012. Anais. . . [S.1.: s.n.], 2012. v. 2011.

[18] SCHUTZBANK A.; FERNANDOPULlE, R. Doubling down: lessons learned from building a new electronic health record as part of primary care practice redesign. Two. ed. São Paulo: Healthcare, 2014.

[19] SIMON S. K.; ANBANANDHEN, S. M. S. L. A Ubiquitous Personal Health Record (uPHR) Framework. In: ACM, 2013. Anais. . [S.1.: s.n.], 2013.

[20] BELYAEV, K.; RAY, I.; RAY, I.; LUCKASEN, G. Personal health record storage on privacy preserving green clouds. In: COLLABORATIVE COMPUTING: NETWORKING, APPLICATIONS AND WORKSHARING (COLLABORATECOM), $2013 \quad 9^{\mathrm{TH}}$ INTERNATIONAL CONFERENCE CONFERENCE ON, 2013. Anais. . [S.1.: s.n.], 2013. p. 448-457.

[21] TENTORI M.; FAVELA, J. Activity-aware computing for healthcare. In: IEEE PERVASIVE COMPUTING, 2008. Anais. . . [S.1.: s.n.], 2008. p. 51-57.

[22] HEDAYAT, R. Semantic web technologies in the quest for compatible distributed health records. In: DISSERTAçãO (DEGREE OF MASTER MATHEMATICS AND COMPUTER SCIENCE) - UPPSALA UNIVERSITY, UPPSALA, 2010. Anais. . [S.1.: s.n.], 2010. p. 65.

[23] COSTA, C. A. da; YAMIN, A. C.; GEYER, C. F. R. Toward a General Software Infrastructure for Ubiquitous Computing. IEEE Pervasive Computing, Piscataway, NJ, USA, v. 7, n. 1, p. 64-73, Jan. 2008.

[24] SANTOS M. R.; BAX, M. P. P. C. Codificando Arquétipos em linguagem ADL com base no modelo de referência da norma ISO 13606. In: 2010. Anais. . [S.1.: s.n.], 2010.

[25] JEONG, H.-Y.; HONG, B.-H. A practical use of learning system using user preference in ubiquitous computing environment. Multimedia Tools Appl., [S.1.], v. 64, n. 2, p. 491-504, 2013.

[26] LIM J.E.; CHOI, O. N. H. B. D. A context-aware fitness guide system for exercise optimization in Uhealth. In: IEEE TRANSACTIONS ON INFORMATION TECHNOLOGY IN BIOMEDICINE: A PUBLICATION OF THE IEEE ENGINEERING IN MEDICINE AND BIOLOGY SOCIETY, 2009. Anais. . . [S.1.: s.n.], 2009. p. 370-379. 
[27] SUDHA, R. e. a. Ubiquitous Semantic Space: a context-aware and coordination middleware for ubiquitous computing. In: IN: INTERNATIONAL CONFERENCE ON COMMUNICATION SYSTEMS SOFTWARE AND MIDDLEWARE, 2007. Anais. . [S.1.: s.n.], 2007. n. 2, p. 1-7.

[28] KINDBERG T.; FOX, A. System software for ubiquitous computing. Pervasive Computing, IEEE, [S.1.], v. 1, n. 1, p. 70-81, 2002.

[29] GOMES, A. R. UbiquitOS - Uma proposta de arquitetura de middleware para adaptabilidade de serviços em sistemas de computação ubíqua. In: 2007. Anais. . . Dissertação de Mestrado em Informática: Universidade Brasília: Brasília, 2007. p. 100.

[30] DEY A. K.; ESTRIN, D. Perspectives on Pervasive Health from Some of the Field's Leading Researchers. In: PERVASIVE COMPUTING, IEEE, 2011. Anais. . [S.1.: s.n.], 2011. p. 2-7.

[31] CHEN G; KOTZ, D. Solar: a pervasive-computing infrastructure for context-aware mobile applications. technical report - tr2002-421. In: 2002. Anais. . [S.1.: s.n.], 2002.

[32] BROWN IAN; ADAMS, A. A. The ethical challenges of ubiquitous healthcare. In: INTERNATIONAL REVIEW OF INFORMATION ETHICS, 2007. Anais. . . [S.1.: s.n.], 2007. p. 54.

[33] CACERES C.; FERNANDEZ, A. O. S. V. M. Agent-Based Semantic Serverice Discovery for Healthcare: an organizational approach. In: INTELLIGENT SYSTEMS, IEEE, 2006. Anais. . . [S.1.: s.n.], 2006. p. $11-$ 20.

[34] NG JOSEPH KEE-YIN; WANG, J. L. K.-Y. K. C. H. C. H. S. Capturing and Analyzing Pervasive Data for SmartHealth. In: INTERNATIONAL CONFERENCE ON ADVANCED INFORMATION NETWORKING AND APPLICATIONS, 28., 2014. Anais. . [S.l.: s.n.], 2014. p. 985-992.

[35] ROGGEN D., A. B.; TROSTER, G. Life Style Management usingWearable Computer. [S.1.]: Proceedings of UbiHealth 2006: The 4th International Workshop on Ubiquitous Computing for Pervasive Healthcare Applications, 2006

[36] KOMNINOS, A.; STAMOU, S. HealthPal: an intelligent personal medical assistant for supporting the selfmonitoring of healthcare in the ageing society. [S.1.]: Proceedings of UbiHealth 2006: The 4th International Workshop on Ubiquitous Computing for Pervasive Healthcare Applications, 2006.

[37] TANTORI M., F. J. Towards the Design of Activity-aware Mobile Adaptive Applications for Hospitals. [S.1.]: Proceedings of Ubi-Health 2006: The 4th International Workshop on Ubiquitous Computing for Pervasive Healthcare Applications, 2006.

[38] TANG, C.; CARPENDALE, S. Healthcare Quality and Information Flow during Shift Change. [S.1.]: Proceedings of UbiHealth 2006: The 4th International Workshop on Ubiquitous Computing for Pervasive Healthcare Applications, 2006.

[39] MASSEY T. GAO, T. B. D. H. A. C. D. W. D. S. L.; SARRAFZADEH, M. Pervasive Triage: towards ubiquitous, real-time monitoring of vital signs for pre-hospital applications. [S.l.]: Proceedings of UbiHealth 2006: The 4th International Workshop on Ubiquitous Computing for Pervasive Healthcare Applications, 2006

[40] FISHKIN K., C. S. R. J. R. B. S. I.; SOUTER, K. Ubiquitous Computing Support for Skills Assessment in Medical School. [S.1.]: Proceedings of UbiHealth 2004: The 3rd International Workshop on Ubiquitous Computing for Pervasive Healthcare Applications, 2004.

[41] MIHAILIDIS A., E. P. G. D. B. J.; HOEY, J. Pervasive Computing to Enable Mobility in Older Adults with Cognitive Impairment. [S.1.]: Proceedings of UbiHealth 2006: The $4^{\text {th }}$ International Workshop on Ubiquitous Computing for Pervasive Healthcare Applications, 2006.

[42] MARI, J. J.; WILLIAMS, P. A validity study of a psychiatric screening questionnaire (SRQ-20) in primary care in the city of Sao Paulo. The British Journal of Psychiatry, [S.1.], v. 148, n. 1, p. 23-26, 1986.

[43] Sistema de Saúde Mãe de Deus. Gerência dos Serviços de Saúde Mental. Avaliação de risco do comportamento. Porto Alegre, 2015.

[44] Likert, R. (1932). A Technique for the Measurement of Attitudes. Archives of Psychology, 140, 1-55.

Revista Brasileira de Computação Aplicada (ISSN 2176-6649), Passo Fundo, v. 9, n. 2, p. 43-58, jul. 201758 